\title{
Paratuberculosis in Camel (Camelus dromedarius): The Diagnostic Efficiency of ELISA and PCR
}

\author{
Ameen M. Alhebabi ${ }^{1,2}$ and Ahmed M. Alluwaimi* ${ }^{* 1,2}$ \\ ${ }^{I}$ Department of Public Health, Military Health Services, King Fahd Health Complex, Damamm, Saudi Arabia \\ ${ }^{2}$ Department of Microbiology and Parasitology, P.O. Box 35252, College of Veterinary Medicine and Animal Resources \\ King Faisal University Al-Ahsaa, 31983, Saudi Arabia
}

\begin{abstract}
The camel paratuberculosis in Saudi Arabia was widely documented. ELISA and PCR were employed to exploit their feasibility for control of the MAP infection in Saudi Arabia. Ruminant ELISA test was used for the screening of 861 serum samples that were collected from three different age groups, 1-4 years (young age), 5-9 years (middle age) and 10-15 years (old age). Only 29 samples were revealed as ELISA positive in which 14 samples were from the young age group. PCR analysis was carried out mainly on the fecal samples that were collected from young age group and all the ELISA positive samples of other groups. PCR detected only 97 positive samples out of 310 tested samples. PCR detected more positive samples in the young age group than ELISA (29\% of 276 young age samples). The ELISA proved effective as a screening tool for MAP infection in camel; however its sensitivity in the young group is skeptical. PCR on the other hand, is effective only as supportive test to the ELISA due to the great variations in the pattern of MAP shedding. Hence, ELISA and PCR were shown feasible for control of the MAP infection, but ELISA was not prudent in the early detection of the infection. Attempt to isolate the camel MAP for its genotyping was not successful despite the incubation for more than 10 weeks.
\end{abstract}

Keywords: Camel, Johne's disease, Saudi Arabia, ELISA, paratuberculosis.

\section{INTRODUCTION}

Dromedary camel (Camelus dromedarius) is one of the highly valuable domestic animals in Saudi Arabia. Camel is multipurpose animal that can be used for meat, milk and wool production. In addition to the previous traditional uses, modern applications in the dairy industry lead to the development of camel dairy farms that are capable of producing camel milk on the commercial level. Camel milk and meat are considered an important source of proteins for wide range of population. Recently, booming of camel racing in the Gulph countries made camel as highly attractive commodity [1].

Mycobacterium avium subspecies paratuberculosis (MAP) causes Johne's disease in domestic and wild ruminant like, cattle, sheep, goats, deer, antelope and bison worldwide [2]. In Saudi Arabia, Johne's disease was reported in sheep, goat, dairy cattle, and camel [3-6].

Long incubation period is the main characteristic feature of MAP infection. Ingestion of fecal material, milk or colostrum is the main route of infection. Infected cattle shed low amount of bacteria during the subclinical stage. However, during the clinical stage the shaded organisms in feces increase dramatically. The major symptoms of infection are chronic diarrhea, emaciation, decrease milk production and infertility [2].

*Address correspondence to this author at the Department of Microbiology and Parasitology, P.O. Box 35252, College of Veterinary Medicine and Animal Resources King Faisal University Al-Ahsaa, 31983, Saudi Arabia; Tel: 966 505935964; Fax: 9663 5816635;

E-mail: alluwaimi@saudivms.org.sa
ELISA remains one of the most convenient tools for the control of MAP infection. Overwhelming studies addressed the sensitivity and specificity of ELISA for detecting the subclinical MAP infection [7-9]. ELISA was developed to detect anti-MAP antibodies in camelid in South America [10]. However, commercially available ELISA was employed to examine its sensitivity for detecting the antiMAP antibodies in camel (Camelus dromedarius) [11]. Screening of 100 camel serum samples at different ages was shown that the bovine ELISA was versatile enough to detect the anti-MAP antibodies. The results reflected the feasibility of the MAP antigen in the ELISA kit for detecting the disease in camel and the practicality of the conjugated antibodies to interact with the camel anti-MAP antibodies [11].

Recent studies clearly demonstrated the influence of the stage of the disease and the nature of the shedding on the early detection of the disease with the ELISA $[8,12,13]$. Using four different ELISA tests, fecal culture and PCR for the early diagnosis of the disease, it was demonstrated that the stage of the incubation period at which the test was performed has great influence on the early diagnosis of the disease. The paratuberculosis incubation period lasts 12-16 weeks in which most of the positive animals are probably detected afterwards [12]. On the other hand, Nielsen (2008) [13] dismissed the strict relation of the positive ELISA to the detection of the organism in feces. It is the nature of the shedding, (intermittent, transitional, low or high shedding) that dictates the number of the shedded organisms. Hence, positive ELISA result in the fecal negative animals was seen quite acceptable [13]. 
Discovery of the distinct insertion segment (IS900) in the MAP genome in the late 1980s has enhanced the development of highly specific and sensitive polymerase chain reaction (PCR) techniques that incorporated the IS900 probe for the early diagnosis of MAP infection [14]. The specificity and sensitivity of PCR system was enhanced further by developing new primers to detect as low as 1 MAP CFU [14]. However, the sensitivity of the PCR remained controversial in comparison to the fecal culture.

The main objective of this study is to examine further the feasibility of ELISA and the PCR as diagnostic tools for MAP control in camel at different ages. The genotyping of the camel (Camelus dromedarius) MAP strain was also attempted.

\section{MATERIALS AND METHODS}

\section{Camel Groups}

The animals were classified into four different groups according to their age. The young age group is 1-4 years old, the middle age group is the 5-9 years old and the late age group is $10-15$ years old.

\section{Samples}

A total of 861 Sera and fecal samples were collected from all the above stated groups. The samples were distributed as, $386(44.83 \%)$ serum and fecal samples from 1-4 years old group, $268(31.12 \%)$ samples from 5-9 years old group and 207 (24.04\%) samples from 10-15 years old.

\section{Detecting Anti-MAP Antibodies with ELISA}

The serum samples were tested by commercial serum paratuberculosis screening ELISA kit (Lsivet Ruminant Serum Paratuberculosis Screening Kit-France). The test was performed according to the manufacturer's directions. Briefly, the serum samples and the controls were adsorbed first against the Mycobacterium phelei antigen and then transferred to the coated plate and incubated at room temperature. After washing to remove all non attached antibodies the mononclonal anti-ruminant antibodies labeled with horse reddish peroxides (HRP) were added and the plate was incubated in the dark for two hours. The conjugated antibodies were then removed and after several washings chromogen substrate was added and incubated. After adding the stop solution the plates were read by ELISA plate reader (Start Fax 2100, Awarness Technology Inc. USA)

\section{Detection of MAP DNA by Real-Time PCR}

Screening for the MAP DNA in the fecal samples was carried out with the real-time PCR kit (VetAlert, Tetracore Johne's Real-Time PCR kit, USA). The kit is made of two parts, the extraction kit and the DNA detection kit. The extraction of the MAP DNA and its detection was performed according to the manufacturer's directions. Briefly, The DNA was extracted using one gram protocol in which one gram of the fecal samples was transferred to $5 \mathrm{ml}$ conical tube and $2 \mathrm{ml}$ of $1 \mathrm{x}$ TE (10mM Tris $\mathrm{HCl}$ and $10 \mathrm{mM}$ EDITA) buffer was added and incubated at room temperature for 5 min. The sample then placed in the disruption tube (containing glass beads) and beadbeating was performed by vortexing. The supernatant was collected after centrifugation at $16000 \mathrm{x} \mathrm{g}$ for $10 \mathrm{~min}$. The procedure of the DNA extraction then performed according to the standard protocol using supplied buffers and spin columns. Finally the DNA was eluted from the column by adding $50 \mu \mathrm{l}$ of $\mathrm{dH} 2 \mathrm{O}$ to the spin column and then incubated at room temperature for 1 min. The DNA was collected by centrifugation at $5,200 \mathrm{xg}$ for 1 minute.

The MAP DNA was detected using MAP DNA real timePCR kit (VetAlert, Tetracore Johne's Real-Time PCR kit, USA). The extracted DNA $(2.5 \mu \mathrm{l})$ or positive control or no template control was mixed with $22.5 \mu 1$ mastermix. The amplification of the MAP DNA was carried out using BioRad IQ optical module thermocycler (BioRad-USA). The run considered valid if the amplification curve of the positive control $(25000$ gene copies $/ 2.5 \mu \mathrm{l})$ reached cycle threshold $(\mathrm{Ct})$ of $20-26$ cycles. The test sample is considered positive if its $\mathrm{Ct}$ is $\leq 38$.

\section{Visualization of the MAP Bacilli with the Zehil-Nielsen Stain}

A fecal smear from some of the fecal samples that recorded high $\mathrm{Ct}$ level was examined directly with the ZehilNielsen staining.

\section{Ante and Postmortem Examination}

The ante and the postmortem profiles of the Johne's disease were applied on selected camels. The antemortem profile was based mainly on emaciation, diarrhea, whereas the postmortem profile was depended on the enlargement of the mesenteric lymph node and the thickness and redness of the ileum.

\section{Fecal Culture and Genotyping}

Efforts to isolate camel MAP strain in and to perform complete genotyping were pursued with the collaboration of Dr. Adel M. Talaat, at the Laboratory of Bacterial Genomics, Department of Pathobiological Sciences, University of Wisconsin, Madison, USA.

\section{RESULTS}

\section{The Feasibility of ELISA Test in Detecting Camel Anti- MAP Antibodies}

Screening of 861 serum samples with the ruminant ELISA test resulted in detection of a total of 29 positive $(3.37 \%)$ and 17 suspected samples (1.97\%) (Table 1). However on the age group level the ELISA positive samples are higher among the age group 1-4 years old (14 out of 386 samples [44.8\%]).

Table 1. ELISA Results of the Entire Serum Samples Grouped According to the Age of Camel

\begin{tabular}{|c|c|c|c|c|}
\hline Age Group & $(+)$ & $(-)$ & $( \pm)$ & Total \\
\hline \hline $1-4$ yrs & $14(1.62 \%)$ & $365(42.3 \%)$ & $7(0.8 \%)$ & $386(44.83 \%)$ \\
\hline $5-9$ yrs & $5(0.58 \%)$ & $262(30.42 \%)$ & $1(0.11 \%)$ & $268(31.12 \%)$ \\
\hline $10-15$ yrs & $10(1.16 \%)$ & $188(21.83 \%)$ & $9(1.04 \%)$ & $207(24.04 \%)$ \\
\hline Total No. & 29 & 815 & 17 & 861 \\
\hline Total \% & $3.37 \%$ & $94.65 \%$ & $1.97 \%$ & $100 \%$ \\
\hline
\end{tabular}


For the age group 5-9 years old the positive samples are only 5 out of 268 serum samples $(0.58 \%)$. Finally, the positive samples are 10 out of 207 (1.16\%) for the age group 10-15 years (Table $\mathbf{1}$ ).

\section{Detection of the MAP DNA with the Real-Time PCR}

Only $310(36 \%)$ fecal samples were selected to be tested by the real-time PCR. The selected samples were the entire ELISA positive samples from the young group (1-4 years old) because of the low sensitivity of ELISA in detecting the disease in this group (early stage of infection) which could result in high ELISA negative output among this age group.

Samples were distributed as follow 1-4 years old (276 samples) (71\%), the 5-9 years old (23 samples) $(8.5 \%)$ and 10-15 years old (11 samples) (5.5\%) (Table 2).

Table 2. The Real-Time PCR Results of the Selected Fecal Samples

\begin{tabular}{|c|c|c|c|}
\hline Age Group & Positive & Negative & Total \\
\hline \hline $1-4$ yrs & $80(29 \%)$ & $196(71 \%)$ & $276(71 \%)$ \\
\hline $5-9$ yrs & $12(52 \%)$ & $11(48 \%)$ & $23(8.5 \%)$ \\
\hline $10-15$ yrs & $5(45 \%)$ & $6(46 \%)$ & $11(5.5 \%)$ \\
\hline Number & 97 & 213 & $310(36 \%)$ \\
\hline Percentage & $31.30 \%$ & $68.70 \%$ & \\
\hline
\end{tabular}

The PCR analysis detected a total of 97 positive samples $(31.30 \%)$. The positive samples that were among the age group 1-4 years old are $80(29 \%)$ (Table 2). The recorded results for the 5-9 years old group are 12 samples $(52 \%)$ and 5 samples (45\%) for the 10-15 years old (Table 2).

\section{The Outcome of the Combined Testing of the Young Age Group with ELISA and PCR}

Due to the low sensitivity of ELISA in detecting all the seroconverted animals in the early stage of the incubation period, it was decided to test with PCR almost all the samples of the age group 1-4 years old with emphasis on the ELISA positive samples. The PCR will confirm the infectious stage of the seroconverted animals as well as the infected stage of the nonseroconverted animals.

The results indicated that only 8 out of 14 ELISApositive samples were PCR positive. Similarly, only 3 samples were PCR positive out of 5 ELISA-positive samples of age group 5-9 years old. However, all the ELISA positive samples of age group 10-15 years old appeared to be PCR positive.

\section{Pre and Postmortem Examination}

Postmortem examination has revealed few camels with obvious clinical signs mainly emaciation which later they appeared to be ELISA and/or PCR positives.

The postmortem examination of ileum and mesenteric lymph nodes of emaciated and other camels indicated no pathological signs typical to Johne's disease.
Ziehl-Neelsen Staining Analysis of Some of the Fecal Smears

Ziehl-Neelsen stained smears from the PCR positive samples showed from moderate to highly condensed numbers of the MAP bacilli.

\section{The Fecal Culture and Genotyping}

Two attempts for culturing the ELISA-PCR positive samples for more than 10 weeks failed to show any tangible growth of MAP colonies. PCR analysis of the slant also failed to detect any growth.

\section{DISCUSSION}

Johne's disease in camel (Camelus dromedarius) in Saudi Arabia is widely documented [6]. Recently the detection of anti-MAP antibodies using bovine ELISA in camel substantiated the possible feasibility of this tool for MAP diagnosis in camel [11].

The application of ELISA and PCR in this study has clearly indicated the influence of age susceptibility in camel to MAP infection. The MAP infectivity was shown to be age dependent [15]. Calves are highly susceptible to the infection than adult cattle even with the minimal infective dose. Although, the ELISA positive samples of age group 1-4 years old were relatively similar to groups of older animals, the retesting with the PCR reconfirmed the limitation of ELISA in detecting all animals that were exposed to the organism. Therefore, limitation of the PCR in revealing all shedding animals and the failure of the ELISA in detecting all seroconverted animals at the young age groups (groups 14 and 5-9 years old) portraits an embedded shortage in the ELISA and PCR as efficient tools for early detection of MAP infection. Hence, ELISA and PCR could play crucial role in monitoring the disease in camel but they are incapable as decisive tool in eradicating MAP in the camel herds in Saudi Arabia. In an interesting approach, Schukken et al. (2009) [16] reviewed the versatility of MAP control and survey programs for the last 20 years in eradicating the MAP infection in the dairy farms. Neither longitudinal survey with ELISA and/or PCR nor fecal culture was effective in the elimination of MAP infection.

The ambiguity of factors that regulate the pattern of MAP shedding and the immunopathological mechanism involved in the initiating the humoral immune responses were seen the major factors that render ELISA and PCR sensitivity variable at the early stage of the incubation period [13]. Monitoring the shedding pattern of large numbers of cattle over 3 years illustrated five types of shedding patterns, nonshedders, transient-shedders, intermittent-shedders, lowshedders and high-shedders, on the basis of number of positive fecal culture and the detected MAP colonies. The results revealed fundamental findings regarding the relation of ELISA results to the pattern of shedding. The most intriguing findings were the possibility of using ELISA as forecasting tool for the commencement of shedding. In most cases ELISA was capable of detecting seroconverted animals before the shedding commenced. However, some ELISA positive animals could remain fecal culture negative while 
others could start shedding before the seroconvertion [13]. Hence, in view of these findings the results of ELISA and PCR presented in this study clearly reflect that PCR has unpredictable value due to the wide variations in the shedding pattern. However, the high numbers of positive ELISA in the age group 1-4 years old definitely support the predictive value of ELISA.

The results of this study also reflected the cross reaction of MAP antigen in the ELISA kit with the camel anti-MAP antibodies and the sensitivity of the conjugated antibodies to detect camel's antibodies. The application of bovine ELISA for detecting anti-MAP in other species like deer was documented $[17,18]$. Similar to our findings, Woodbury et al. (2008) [18] experienced low ELISA sensitivity in detecting of anti-MAP antibodies in the early stage of infection. Nevertheless, fecal cultures and culture slant-PCR has confirmed the ELISA positive results. To optimize the ELISA sensitivity for MAP detection in deer, Tryland et al. (2004) [17] compared the sensitivity of preadsorbed commercial ELISA with that of ELISA equipped with conjugated protein-G instead of anti-species conjugated antibodies. However, extensive testing with both ELISA tests revealed minimal differences in their sensitivities. In general, despite the controversies about the antigen specificity and the versatility of the conjugated anti-species antibodies, ELISA remains a useful screening test in control programs rather than a sensitive test for the early detection of the infection.

\section{CONCLUSION}

The study has reconfirmed the spread of MAP infection in the Saudi camel herds. The ruminant ELISA was proved useful for the screening of the MAP infection in camel and could rule out the need for the species specific ELISA test. ELISA and PCR have shown their feasibility as robust diagnostic tool for screening of Johne's disease in camel. However, their efficiency as useful tools in the longitudinal control programs in the Saudi camel herds requires further investigation. Although, PCR proved more practical than ELISA in detecting MAP, the different shedding patterns render PCR of low predictive value unless it is employed in combination with milk or serum ELISA.

\section{ACKNOWLEDGEMENT}

This study was supported by a grant from SABIC, the Saudi petrochemical industrial company.

\section{REFERENCES}

[1] Breulmann M, Böer B, Wernery U. et al. The Camel from tradition to modern times. UNESCO Office, Doha, Qatar 2007.

[2] Stabel JR, John's disease: A hidden threat. J Dairy Sci 1997; 81: 283-8.

[3] Ahmed SA, Towfik A. Johne's disease among sheep and goat of El-Qassiem area. 16th Annual meeting of Saudi Biological society, 1995.

[4] Al Hajri SM, Alluwaimi AM. ELISA and PCR for evaluation of subclinical paratuberculosis in the Saudi dairy herds. Vet Microbiol 2007; 121: 384-5.

[5] Alluwaimi AM, Hatem ME, Almousa JM. The efficacy of gel immunodiffusion and fecal smear tests for diagnosis of ovine paratuberculosis in sheep in Saudi Arabia. Egyp J Immunol 1999; 7: 29-32.

[6] Gameel AA, Ali AS, Razig SA, et al. A clinico-pathological study on spontaneous paratuberculosis in camels (Camelus dromedarius). Pak Vet J 1994; 14: 15-19.

[7] Weber MF. Surveillance of Mycobacterium avium subsp. Paratuberculosis in dairy herds. The paratuberculosis Newsletter, 2010; March: 5-14.

[8] Collins MT, Scott JW, Petrini et al. Evaluation of five antibody detection tests for diagnosis of bovine paratuberculosis. Clin Dig Lab Immunol 2005; 12: 685-92.

[9] Nielsen SS, Toft N. Age-specific characteristics of ELISA and fecal culture for purpose-specific testing for paratuberculosis. J Dairy Sci 2006; 89: 569-79.

[10] Kramsky JA, Miller DS, Hope A, et al. Modification of a bovine ELISA to detect camelid antibodies to Mycobacterium paratuberculosis. Vet Microbiol 2000; 77: 333-7.

[11] Alluwaimi AM. The efficiency of bovine ELISA in detection of the Mycobacterium avium subspecies paratuberculosis (MAP) infection in camel (Camelus dromedaries) at different ages. J. Camel Pract Res 2008; 15: 163-5.

[12] Diéguez FJ, González AM, Menéndez S, et al. Evaluation of four commercial serum ELISAs for detection of Mycobacterium avium subsp. paratuberculosis infection in dairy cows. Vet J 2009; 180: 231-5

[13] Nielsen SS. Transitions in diagnostic tests used for detection of Mycobacterium avium subsp. Paratuberculosis infections in cattle. Vet Microbiol 2008; 132: 274-82.

[14] Vansnick E, Rijk PD, Vercammen F, et al. Newly developed primers for the detection of Mycobacterium avium subspecies paratuberculosis. Vet Microbiol 2004; 100: 197-204.

[15] Windsor PA, Whittington RJ. Evidence for age susceptibility of cattle to Johne's disease. Vet J 2010; 184: 37-44.

[16] Schukken YH, Mitchell RM, Pradhan Lu, et al. Elimination of Mycobacterium avium subspecies paratuberculosis from dairy farms: fact or fiction? Proceedings of $10^{\text {th }}$ International Colloquium of Paratuberculosis. 2009; 109-13.

[17] Tryland M, Olsen I, Vikøren T, et al. Serologic survey for antibodies against Mycobacterium avium subsp. paratuberculosis in free-ranging cervids from Norway. Wild L Dis 2004; 40: 32-41.

[18] Woodbury MR, Chirino-Trejo M, Mihajlovic B. Diagnostic detection methods for Mycobacterium avium subsp. Paratuberculosis in white-tailed deer. Cand Vet J 2008; 49: 683-8. 